\title{
BMJ Open Establishment of sex-specific reference intervals for automated haematology analyser-delivered research parameters in healthy Korean adults: a retrospective database review
}

\author{
Kibum Jeon, ${ }^{1}$ Miyoung Kim (10 , ${ }^{2}$ Junhee Han, ${ }^{3}$ Jiwon Lee, ${ }^{2}$ Jee-soo Lee, ${ }^{2}$ \\ Han-Sung Kim, ${ }^{2}$ Hee Jung Kang, ${ }^{2}$ Young Kyung Lee ${ }^{2}$
}

To cite: Jeon K, Kim M, Han J, et al. Establishment of sexspecific reference intervals for automated haematology analyser-delivered research parameters in healthy Korean adults: a retrospective database review. BMJ Open 2020;10:e036887. doi:10.1136/ bmjopen-2020-036887

- Prepublication history and additional material for this paper is available online. To view these files, please visit the journal online (http://dx.doi.org/10. 1136/bmjopen-2020-036887)

Received 13 January 2020 Revised 14 August 2020 Accepted 30 August 2020

\section{Check for updates}

(C) Author(s) (or their employer(s)) 2020. Re-use permitted under CC BY-NC. No commercial re-use. See rights and permissions. Published by BMJ.

${ }^{1}$ Department of Laboratory Medicine, Hangang Sacred Heart Hospital, Seoul, Republic of Korea

${ }^{2}$ Department of Laboratory Medicine, Hallym University Sacred Heart Hospital, Anyang, Gyeonggi-do, The Republic of Korea

${ }^{3}$ Department of Statistics, Hallym University, Chuncheon, Gangwon, The Republic of Korea

Correspondence to Professor Miyoung Kim; rabbit790622@gmail.com

\section{ABSTRACT}

Objectives Automated haematology analysers measure various parameters of relevance to clinical research along with routine complete blood count (CBC)-related components. We aimed to establish ethnicity-specific and sex-specific reference intervals for 26 research-specific parameters as well as 18 routinely reported components using a large cohort of healthy Korean adults. The necessity of requiring separate sex-specific reference intervals for each parameter was also examined.

Design A retrospective database review.

Setting Single tertiary-care hospital of approximately 375 physicians and 530 nurses.

Participants This study included 1383 reference

individuals (840 men and 543 women).

Primary and secondary outcome measures Following the Clinical and Laboratory Standards Institute guidelines for establishing reference intervals, routine CBCs as well as research parameters were measured using an ADVIA 2120i instrument.

Results All the routine components except for mean platelet volume and per cent lymphocytes differed significantly between men and women. Most research parameters also differed between the sexes; the exceptions were large platelets, platelet dry mass distribution width, per cent basophil saturation, per cent peroxidase saturation and per cent abnormal peroxidase absorption. Despite these differences, separate reference intervals for men and women were required only for two research-specific parameters: 'percentage high cellular haemoglobin' and 'percentage of hyperchromic red blood cells (RBCs)'.

Conclusion Even though most parameters showed significant differences between men and women, none of the evaluated parameters except two RBC-related factors required separate reference intervals for each sex.

\section{INTRODUCTION}

The clinical decision-making process begins by comparing data obtained from a patient to reference values. ${ }^{1}$ To reliably interpret test results, accurate reference intervals that are
Strengths and limitations of this study

- A set of stringent criteria were used to select the healthy individuals for this study (although not all CLSI guideline criteria could be evaluated).

- The study is the largest of its kind to date.

The study also examined the necessity of establishing sex-specific reference intervals.

- Our exclusion criteria did not incorporate all the rules mentioned in the CLSI guideline, but included additional rules to best exclude non-healthy individuals.

- We were unable to evaluate some other parameters that were found to be clinically significant in previous studies because limited data availability.

derived from healthy individuals and categorised according to major covariates such as age, sex and/or ethnicity are required. An important step in establishing accurate reference intervals is selecting a sufficiently large number of healthy reference individuals representing relevant demographic groups. ${ }^{1-4}$ Achieving this requires establishing and applying a set of criteria to exclude non-healthy individuals from the reference population. 1

Modern automated haematology analysers use up-to-date techniques including electrical impedance, radiofrequency conductivity, light scattering and/or cytochemistry. ${ }^{5}$ They enable the gathering not only of data that are routinely reported to patients but also various research parameters that deliver valuable clinical information. ${ }^{67}$ Such parameters include those that reflect the size and haemoglobinisation of red blood cells $(\mathrm{RBCs})^{8}$ as well as the morphological parameters (cell size, cytoplasmic granularity and nuclear lobularity) of white blood cells (WBCs). ${ }^{5}$ Platelet-related parameters include the distribution width, 
plateletcrit (PCT), mean platelet component, mean platelet mass (MPM) and large platelet count. ${ }^{9}$

Previous representative studies found that certain research parameters have particularly useful clinical implications in practice. Kim et al showed that platelet indices including PCT, platelet component distribution width (PCDW), platelet dry mass distribution width (PMDW) and mean platelet volume (MPV) were useful for predicting the in-hospital mortality of patients suspected of having disseminated intravascular coagulation. ${ }^{10}$ Another study suggested that the reticulocyte haemoglobin content $(\mathrm{CHr})$ correlated well with the Ferritin Index and was a useful marker of functional iron deficiency in blood donors. ${ }^{8}$ In a study that validated an algorithm-guided preoperative anaemia management method that raises the perioperative haemoglobin level and reduces blood transfusion volume, Enko et al used the $\mathrm{CHr}$ to define functional iron deficiency in their study subjects. ${ }^{11}$ Furthermore, Rocco et al detected acute promyelocytic leukaemia by combining six haematologic parameters including large unstained cells (LUCs), hyperchromic cells, per cent saturated cells, platelets, monocyte percentage and blast percentage. ${ }^{12}$ Such research parameters as well as others would be more applicable to real-world practice if reliable reference intervals are provided for each.

Because there are no published research parameter reference intervals based on healthy adult Koreans to date, we aimed to establish appropriate sex-specific reference intervals for 26 research parameters as well as for 18 routinely reported complete blood count $(\mathrm{CBC})$ components. We also performed a comparative analysis of all these parameters in healthy Korean men and women to investigate the necessity of establishing separate reference intervals for each sex. Importantly, we selected a large healthy population based on a set of stringent criteria to ensure high-quality test results.

\section{MATERIALS AND METHODS \\ Selection of healthy reference individuals}

An indirect sampling technique was used to select healthy reference individuals; ${ }^{1}$ the process is depicted in figure 1 . The study initially recruited 8936 participants (4914 men and 4022 women) targeting those between 20 and 60 years of age (mean for total cohort: 46.0 years, range: 20.0-60.0 years; mean for men: 46.6 years, range: 20.0-60.0 years; and mean for women: 45.6 years, range: 20.0-60.0 years) who underwent regular health check-ups at Hallym University Sacred Heart Hospital, Republic of Korea, between September 2014 and April 2018 and whose CBCs were obtained using the ADVIA 2120i instrument (Siemens, Munich, Germany).

Among these subjects, we selected only those who underwent electrocardiography, chest radiography, abdominal ultrasonography and laboratory tests that included CBC, chemistry, viral serology and urinalysis. During this process, 7264 participants (3919 men and 3345 women comprising $79.8 \%$ and $83.2 \%$ of the parent populations, respectively) were excluded owing to the lack of one or

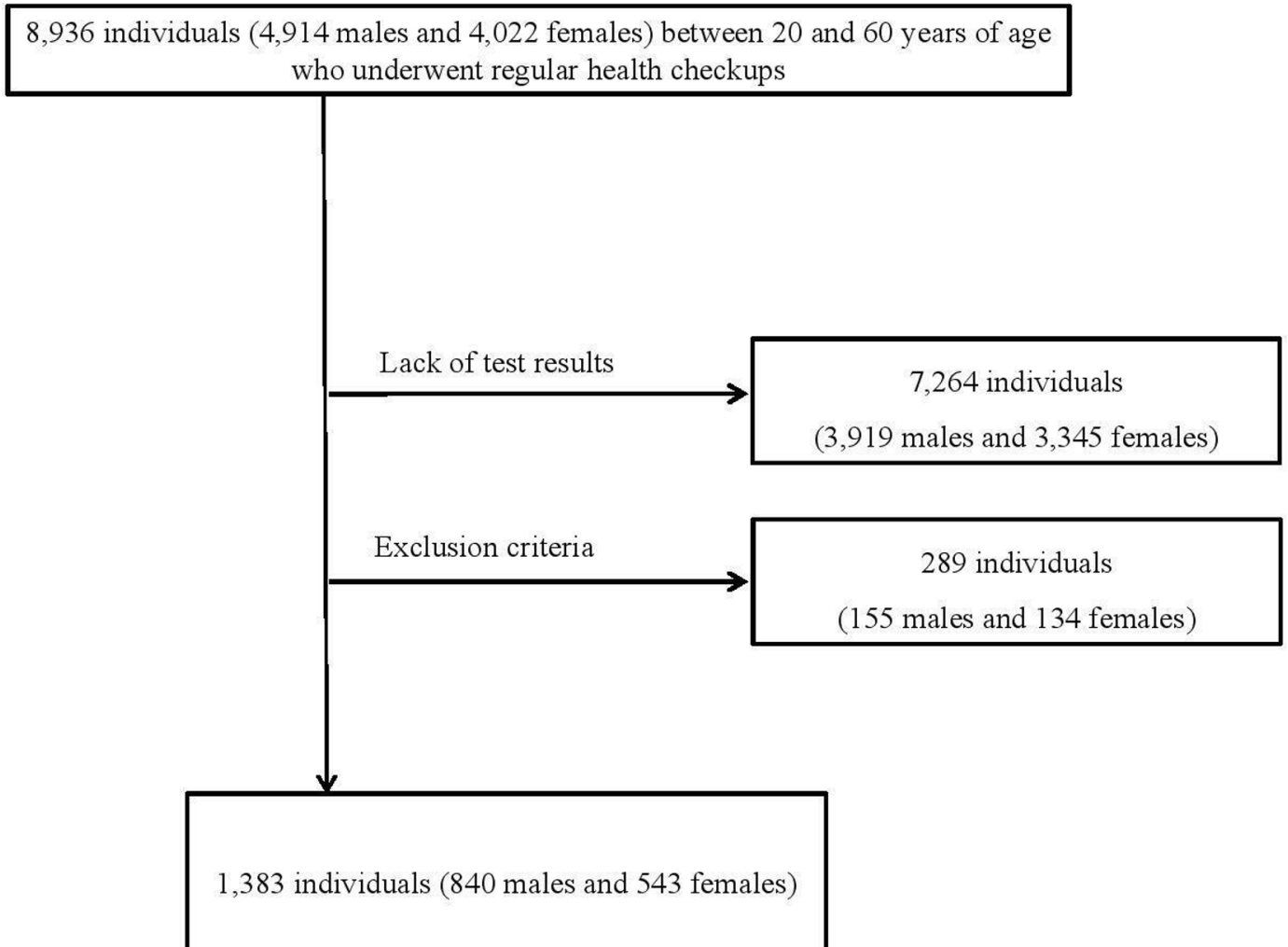

Figure 1 Schematic illustration of the selection process for reference individuals. 
Box 1 Exclusion criteria applied for selecting healthy reference individuals from the parent population

Laboratory
Hyperlipidaemia (low-density lipoprotein $>3.362 \mathrm{mmol} / \mathrm{L}$ ), diabetes
mellitus (fasting blood glucose $>5.55 \mathrm{mmol} / \mathrm{L}$ or glycated haemoglobin
$>7.8 \mathrm{mmol} / \mathrm{L}$ )
Positivity for hepatitis B surface antigen and/or hepatitis $\mathrm{C}$ virus antibody
Abnormal urinalysis results, including the presence of erythrocytes,
granulocytes, glucose, protein or nitrite
Serum iron level $<6 \mu \mathrm{mol} / \mathrm{L}$
Haemoglobin concentration $<90 \mathrm{~g} / \mathrm{L}$
White blood cells $<3.0 \times 10^{9} / \mathrm{L}$ or $>12.5 \times 10^{9} / \mathrm{L}$
Electrocardiography
Abnormal findings on electrocardiography, including ST segment eleva-
tion, pacemaker insertion or evidence of ischaemic heart disease
Imaging studies
Abnormal findings in chest radiography, including suspected thyroid
disease, mediastinal tumortumour, active tuberculosis, pneumonia or
lung cancer
Abnormal findings in abdominal ultrasonography, including suspected
alcoholic liver disease or liver cirrhosis

more required test results. Next, we applied exclusion criteria to extract non-healthy individuals (box 1); these were devised based on the Clinical and Laboratory Standards Institute (CLSI) EP28-A3 guideline as well as additional measures. ${ }^{1}$ Finally, 1383 reference individuals $(840$ men and 543 women representing $17.1 \%$ and $13.5 \%$ of their parent populations, respectively) were selected. The median age of all subjects in the final cohort was 48.0 years (range: $23.0-60.0$ years); that of men was 51.0 years (range: 23.0-60.0 years), while that of women was 45.0 years (range: $24.0-60.0$ years).

\section{Sample collection and measurement of parameters}

Venous whole blood $(5 \mathrm{~mL})$ was drawn from the cubital vein into a K2-ethylenediaminetetraacetic acid (EDTA) tube (Becton Dickinson, Franklin Lakes, New Jersey, USA) after fasting for at least 8 hours prior to phlebotomy. The tube was inverted five to six times and transported to the haematology laboratory.

An ADVIA 2120i instrument was used to measure 18 routine $\mathrm{CBC}$ parameters and 26 research parameters, according to the manufacturer's instructions, within 4 hours of sample collection. The 18 routine parameters included RBC count, haemoglobin, haematocrit (Hct), mean corpuscular volume (MCV), mean corpuscular haemoglobin $(\mathrm{MCH}), \mathrm{MCH}$ concentration $(\mathrm{MCHC})$, red cell distribution width, WBC, neutrophil count, neutrophil fraction, lymphocyte fraction, monocyte fraction, eosinophil fraction, basophil fraction, LUC fraction, platelets, MPV and platelet distribution width (PDW).

We included 26 research parameters (12 RBC, 7 platelet, 3 basophil and 4 peroxisome) based on the data availability and usefulness as described in the previous studies. ${ }^{9} 1314$ RBC parameters included cellular haemoglobin $(\mathrm{CH})$,
$\mathrm{CH}$ concentration mean, per cent high $\mathrm{CH}$, per cent low $\mathrm{CH}, \mathrm{CH}$ distribution width, percentage of hyperchromic RBCs (\%Hyper), percentage of hypochromic RBCs, percentage of macrocytic RBCs, percentage of microcytic RBCs, RBC volume and haemoglobin concentration covariance, $\mathrm{R}$ count (red cell count as measured in a different channel from that used to obtain the routine parameter RBC count) and haemoglobin distribution width. Peroxidase-related parameters included the Mean Peroxidase Staining Index (MPXI), percentage of peroxidase saturation (Perox \%Saturation), percentage of neutrophils with a high absorption value and percentage of abnormal peroxidase absorption (Perox \%Abnormal). Basophil-related parameters included the Lobularity Index, percentage of polymorphonucleated neutrophils (which is distinct from the routinely measured neutrophil fraction) and percentage of basophil saturation (Baso \%Saturation). Platelet parameters included large platelets, mean platelet component, MPM, two-dimensional platelet count, PCDW, PMDW and PCT.

\section{Statistical analysis}

Statistical analyses for the comparison of values between the two sex group, the establishment of reference intervals for each group and the necessity for separate reference intervals were performed as described in our previous study; ${ }^{15}$ namely, differences in values between the two groups were compared using the Mann-Whitney U test. P values $<0.05$ were considered statistically significant.

The reference intervals for measured parameters were established according to a non-parametric method based on the CLSI EP28A-3C criteria for each sex group. ${ }^{1}$ The lower and upper reference limits (the values of the 2.5th and 97.5th percentiles, respectively) for each parameter were established, and the $90 \%$ CIs for the upper and lower limits of each reference interval were determined as follows;

$$
\text { percentile }= \pm 2.81 \frac{S_{y}}{\sqrt{n}}
$$

where $\mathrm{S}$ is the $\mathrm{SD}$ of the reference values and $\mathrm{n}$ is the number of values. ${ }^{16}$ Reed et al suggest a cut-off value of $1 / 3$; that is, if the observed value of $\mathrm{D}$ (the absolute difference between an extreme observation and the next largest (or smallest) observation) was equal to or greater than onethird of the range $\mathrm{R}$ (range of all observations, including extremes), the extreme observation would be deleted. This method was used to exclude extreme outliers. ${ }^{17}$ To determine whether separate reference intervals for each sex group were necessary, we used the method of Harris and Boyd, ${ }^{118}$ who suggested criteria based on the ratio between subclass $\mathrm{SD}$, a normal deviate test of means and calculation of critical decision values dependent on the sample size. ${ }^{19}$ It recommends partitioning reference intervals for different groups in the following situations: (1) the standard normal deviation ( $\mathrm{z}$ ) exceeds the critical value $\left(\mathrm{z}^{*}\right)$, which is $3\left(\mathrm{n}_{\text {average }} / 120\right)^{1 / 2}$, where $\mathrm{n}$ is the number of individuals in the subgroup; (2) the larger 
standard deviation (s2) exceeds 1.5-fold the smaller standard deviation (s1), or equivalently, s2/(s2-s1) is less than 3 regardless of the $\mathrm{z}$ value. If these conditions are met, separate reference intervals should be calculated for each subclass assuming that the difference between the two reference intervals is likely to be of clinical importance. ${ }^{1}$ Data were analysed using the Statistical Package for Social Sciences V.24.0 (IBM Corp., Armonk, New York, USA) and MedCalc V.17.9.7 (MedCalc Software, Ostend, Belgium).

\section{Patient/public involvement}

There were no patients involved in this research.

\section{RESULTS}

\section{Reference intervals for 18 routine parameters}

The calculated median (IQRs) and reference intervals with $90 \%$ CIs for the upper and lower limits of the reference intervals of 18 routine CBC parameters in each sex group are presented in table 1 ; histograms are also shown in online supplemental figure 1 .

Among 18 routinely reported CBC parameters, 16 were significantly different in terms of median and IQR values between the 2 sexes. RBC, haemoglobin, Hct, MCV, MCH, MCHC, PDW, WBC, neutrophil count, monocyte fraction, eosinophil fraction, basophil fraction and LUC fraction were significantly higher in men than in women (all $p$ values were $<0.001$ except for Hct, which was 0.003 ). Platelets, red cell distribution width and neutrophil fraction were significantly higher in women than in men ( $p<0.001,0.005$ and $<0.001$, respectively). There was no significant difference in MPV and lymphocyte fraction between the two groups $(p>0.05)$. None of the 18 parameters required separate reference intervals for each sex.

\section{Reference intervals for $\mathbf{2 6}$ research parameters}

The calculated median (IQR) and reference intervals with $90 \%$ CIs for the upper and lower limits of reference intervals, as well as results of the analyses of whether separate reference intervals are required for each sex, are summarised in table 2; histograms are also shown in online supplemental figure 2.

Among the peroxidase-related parameters, the percentage of neutrophils with a high absorption value was significantly higher in men than in women $(p=0.005)$, while MPXI was significantly higher in women than in men $(\mathrm{p}=0.015)$. Perox \%Saturation and Perox \%Abnormal did not show any significant differences between the two groups (all $\mathrm{p}>0.05$ ). Among the basophil-related parameters, Lobularity Index and the percentage of polymorphonucleated neutrophils were significantly higher in women than in men (all $\mathrm{p}<0.001)$. Baso \% Saturation did not show any statistically significant difference between the two groups $(\mathrm{p}>0.05)$. Among RBC parameters, $\mathrm{CH}$, $\mathrm{CH}$ concentration mean, \%High, $\mathrm{CH}$ distribution width, \%Hyper, percentage of macrocytic RBCs and red cell count were significantly higher in men than in women (all $\mathrm{p}<0.001$ ), while per cent low $\mathrm{CH}$, percentage of hypochromic RBCs, percentage of microcytic RBCs and RBC haemoglobin concentration covariance were significantly higher in women than in men (all $\mathrm{p}<0.001)$. Among platelet-related parameters, PCDW was significantly higher in men than in women $(\mathrm{p}<0.001)$, while the mean platelet component, MPM, two-dimensional platelet count and PCT were significantly higher in women than in men (all $\mathrm{p}<0.001$ except for that of MPM, which was 0.004). Large platelet counts and PMDW were not significantly different between the two groups ( $p>0.05)$. Notably, separate reference intervals for the two sex groups were required for \%High and \%Hyper.

\section{DISCUSSION}

We established reference intervals for 26 research-related parameters as well as 18 routinely obtained CBC components for healthy Korean adults. The healthy subjects were outpatients who voluntarily underwent medical check-ups at private facilities (at their own expense) that offer more extensive examinations than those provided under the national health check-up system. Therefore, we were able to obtain additional health status information that was helpful for selecting a healthy cohort. Our data showed that, even though many parameters were significantly different between the two sex groups, separate reference intervals were not required for most such parameters that we evaluated. When the values of 18 routine parameters were compared between the sexes, all except MPV and lymphocyte fraction showed significant differences, which was likely owing to the physiologic differences between the sexes and/or the large number of values $(\mathrm{N})$ included.

A notable finding was that none of the 18 routine CBC parameters required separate reference intervals for each sex even though sex-specific reference intervals are generally used for routine CBC parameters in clinical laboratories. Our results suggest that clinicians and laboratory personnel may be able to use a common set of reference intervals for both men and women. However, additional studies of populations of varying ethnicities as well as of other haematology analyser brands would help clarify this issue.

Almost all of the $26 \mathrm{CBC}$ research parameters we examined showed significant differences between the sexes; the exceptions included large platelets, PMDW, Baso \% Saturation, Perox \% Saturation and Perox \%Abnormal. As mentioned above, this phenomenon might be a consequence of physiologic differences between men and women or of the large number of reference individuals used in our study. Only a few studies that established reference intervals for research parameters used the same instrument as ours; however, only 2 of the 26 parameters (per cent high $\mathrm{CH}$ and \%Hyper) required separate reference intervals for each sex. These parameters are indicators of haemoglobin concentration and RBC size; thus, interpreting this result should take into 
Table 1 Age-specific and sex-specific medians, IQRs and reference intervals for 18 complete blood count components obtained from healthy Korean adults

\begin{tabular}{|c|c|c|c|c|c|c|c|c|}
\hline Parameter & Sex & $\mathbf{N}$ & Median & IQR & $P$ value & $\begin{array}{l}\text { Reference } \\
\text { interval }\end{array}$ & Lower $90 \% \mathrm{Cl}$ & Upper $90 \% \mathrm{Cl}$ \\
\hline \multirow[t]{2}{*}{$\mathrm{RBC}, \times 10^{12} / \mathrm{L}$} & M & 840 & 4.9 & $4.7-5.1$ & $<0.001$ & $4.3-5.4$ & 4.3 to 4.4 & 5.4 to 5.4 \\
\hline & $\mathrm{F}$ & 543 & 4.3 & $4.1-4.5$ & & $3.8-4.8$ & 3.8 to 3.9 & 4.7 to 4.8 \\
\hline \multirow[t]{2}{*}{$\mathrm{Hb}, \mathrm{g} / \mathrm{L}$} & M & 840 & 152 & $146-158$ & $<0.001$ & $137-167$ & 135 to 138 & 166 to 169 \\
\hline & $\mathrm{F}$ & 543 & 131 & $125-137$ & & $115-144$ & 113 to 117 & 143 to 144 \\
\hline \multirow[t]{2}{*}{ Hct, L/L } & M & 840 & 0.442 & $0.421-0.458$ & 0.003 & $0.397-0.488$ & 0.395 to 0.401 & 0.483 to 0.492 \\
\hline & $\mathrm{F}$ & 543 & 0.387 & $0.370-0.401$ & & $0.346-0.426$ & 0.343 to 0.349 & 0.423 to 0.428 \\
\hline \multirow[t]{2}{*}{ MCV, fL } & M & 840 & 90.6 & $88.1-93.3$ & $<0.001$ & $84.7-96.9$ & 84.3 to 85.1 & 96.4 to 97.3 \\
\hline & $\mathrm{F}$ & 543 & 90.0 & $87.5-92.3$ & & 82.9-95.5 & 81.9 to 83.9 & 95.0 to 96.3 \\
\hline \multirow[t]{2}{*}{$\mathrm{MCH}, \mathrm{fmol}$} & M & 840 & 1.936 & $1.880-1.992$ & $<0.001$ & $1.812-2.073$ & 1.794 to 1.818 & 2.067 to 2.091 \\
\hline & $\mathrm{F}$ & 543 & 1.887 & $1.831-1.949$ & & $1.719-2.017$ & 1.707 to 1.738 & 2.005 to 2.029 \\
\hline \multirow[t]{2}{*}{$\mathrm{MCHC}, \mathrm{g} / \mathrm{L}$} & M & 840 & 344 & $337-352$ & $<0.001$ & $327-361$ & 326 to 328 & 361 to 363 \\
\hline & $\mathrm{F}$ & 543 & 339 & $331-345$ & & $320-356$ & 319 to 321 & 355 to 357 \\
\hline \multirow{2}{*}{$\begin{array}{l}\text { RDW } \\
\text { (proportion of } 1.0 \text { ) }\end{array}$} & M & 840 & 0.125 & $0.123-0.129$ & 0.005 & $0.118-0.134$ & 0.118 to 0.119 & 0.133 to 0.135 \\
\hline & $\mathrm{F}$ & 543 & 0.126 & $0.122-0.131$ & & $0.117-0.142$ & 0.117 to 0.118 & 0.140 to 0.145 \\
\hline \multirow[t]{2}{*}{ WBC, $\times 10^{9} / \mathrm{L}$} & $M$ & 840 & 5.7 & $4.9-6.8$ & $<0.001$ & $3.9-8.9$ & 3.7 to 4.0 & 8.6 to 9.2 \\
\hline & $\mathrm{F}$ & 543 & 5.2 & $4.9-6.2$ & & $3.6-8.2$ & 3.5 to 3.7 & 7.9 to 8.8 \\
\hline \multirow{2}{*}{$\begin{array}{l}\text { Neutrophil count, } \\
\times 10^{9} / \mathrm{L}\end{array}$} & M & 840 & 3.1 & $2.5-3.9$ & 0.001 & $1.5-6.6$ & 1.5 to 1.6 & 6.2 to 7.5 \\
\hline & $\mathrm{F}$ & 543 & 2.9 & $2.3-3.7$ & & $1.6-5.9$ & 1.4 to 1.7 & 5.5 to 6.8 \\
\hline \multirow{2}{*}{$\begin{array}{l}\text { Neutrophil } \\
\text { (fraction) }\end{array}$} & M & 840 & 0.551 & $0.493-0.607$ & $<0.001$ & $0.363-0.729$ & 0.351 to 0.383 & 0.709 to 0.751 \\
\hline & $\mathrm{F}$ & 543 & 0.569 & $0.514-0.623$ & & $0.412-0.726$ & 0.384 to 0.422 & 0.713 to 0.760 \\
\hline \multirow{2}{*}{$\begin{array}{l}\text { Lymphocyte } \\
\text { (fraction) }\end{array}$} & M & 840 & 0.329 & $0.279-0.387$ & 0.662 & $0.180-0.480$ & 0.140 to 0.190 & 0.478 to 0.500 \\
\hline & $\mathrm{F}$ & 543 & 0.331 & $0.281-0.379$ & & $0.188-0.477$ & 0.149 to 0.203 & 0.458 to 0.491 \\
\hline \multirow{2}{*}{$\begin{array}{l}\text { Monocyte } \\
\text { (fraction) }\end{array}$} & $M$ & 840 & 0.055 & $0.047-0.063$ & $<0.001$ & $0.034-0.081$ & 0.033 to 0.037 & 0.079 to 0.087 \\
\hline & $\mathrm{F}$ & 543 & 0.049 & $0.042-0.058$ & & $0.031-0.078$ & 0.029 to 0.033 & 0.075 to 0.082 \\
\hline \multirow{2}{*}{$\begin{array}{l}\text { Eosinophil } \\
\text { (fraction) }\end{array}$} & M & 840 & 0.027 & $0.017-0.042$ & $<0.001$ & $0.006-0.088$ & 0.005 to 0.007 & 0.082 to 0.095 \\
\hline & $\mathrm{F}$ & 543 & 0.021 & $0.014-0.031$ & & $0.003-0.069$ & 0.004 to 0.006 & 0.066 to 0.081 \\
\hline \multirow[t]{2}{*}{ Basophil (fraction) } & $M$ & 840 & 0.004 & $0.003-0.006$ & $<0.001$ & $0.001-0.010$ & 0.001 to 0.001 & 0.010 to 0.010 \\
\hline & $\mathrm{F}$ & 543 & 0.004 & $0.003-0.005$ & & $0.001-0.009$ & 0.001 to 0.001 & 0.008 to 0.011 \\
\hline \multirow[t]{2}{*}{ LUC (fraction) } & M & 840 & 0.020 & $0.016-0.025$ & $<0.001$ & $0.012-0.032$ & 0.011 to 0.012 & 0.031 to 0.035 \\
\hline & $\mathrm{F}$ & 543 & 0.019 & $0.015-0.023$ & & $0.011-0.030$ & 0.010 to 0.012 & 0.029 to 0.032 \\
\hline \multirow[t]{2}{*}{ PLT, $\times 10^{9} / \mathrm{L}$} & M & 840 & 233 & 205.0-263.0 & $<0.001$ & $163.0-324.0$ & 161.0 to 167.0 & 318.0 to 331.0 \\
\hline & $\mathrm{F}$ & 543 & 244 & $211.5-277.5$ & & $176.0-336.8$ & 169.0 to 179.0 & 325.0 to 352.0 \\
\hline \multirow[t]{2}{*}{ MPV, fL } & $M$ & 840 & 8.1 & $7.6-8.6$ & 0.190 & $7.1-9.5$ & 7.0 to 7.1 & 9.3 to 9.6 \\
\hline & $\mathrm{F}$ & 543 & 8.0 & $7.6-8.5$ & & 7.2-9.4 & 7.1 to 7.2 & 9.2 to 9.5 \\
\hline
\end{tabular}

$\mathrm{Hb}$, haemoglobin; Hct, haematocrit; LUC, large unstained cell; MCH, mean corpuscular haemoglobin; MCHC, mean corpuscular haemoglobin concentration; MCV, mean corpuscular volume; MPV, mean platelet volume; PLT, platelets; RBC, red blood cells; RDW, red cell distribution width; WBC, white blood cell.

consideration the fact that MCV and MCHC did not require separate reference intervals for each sex. Red cell counts and haemoglobin levels in women are significantly lower than those in men; this is known to be of clinical significance. We used Harris and Boyd's method to determine whether separate reference intervals are required for men and women; however, the reasons for these well-known difference are not fully clear, and further discussion is needed.

A few studies have investigated reference intervals for research parameters using the ADVIA 2120i, most of which established such reference intervals for particular parameters: Nikulshin et al reported a reference interval for MPXI in children, ${ }^{13} \mathrm{Oh}$ et al did the same for MPXI 


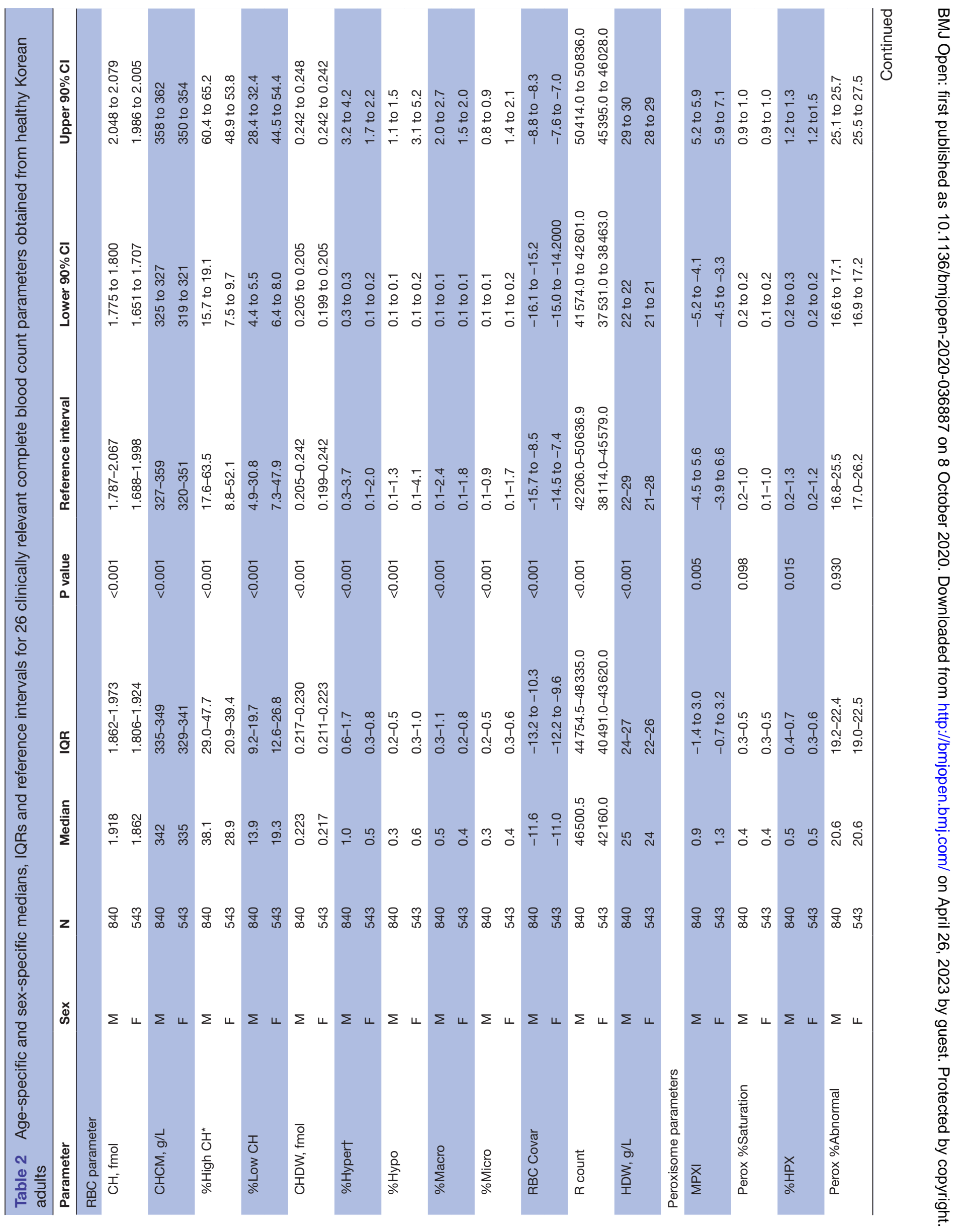




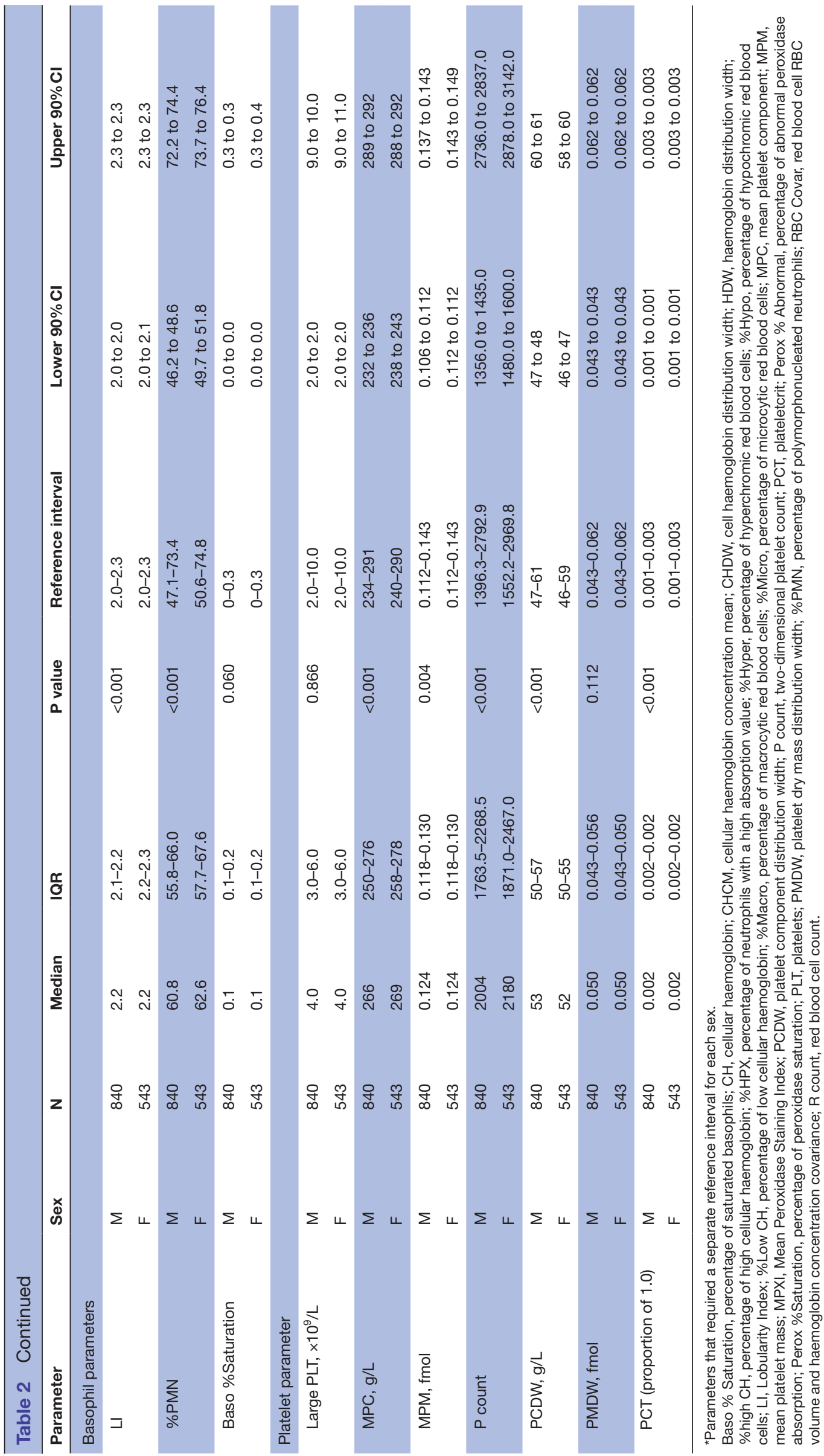


in adults ${ }^{14}$ and Kim et al reported reference intervals for platelet parameters in a study of subjects $\geq 50$ years of age. ${ }^{9}$ The MPIX was significantly higher in women than in men (1.3 (-0.7 to 3.2$)$ and 0.9 (-1.4 to 3.0$)$, respectively; $p=0.015$ ) in our study, these were comparable with the values obtained by $\mathrm{Oh}$ et al even though they did not directly compare the two sex groups. ${ }^{14}$ As they also did not investigate the necessity of separate reference intervals for each sex, we could not compare our findings with theirs. We also could not directly compare our results concerning platelet parameters with those of Kim et at because they limited their reference individuals to subjects over 50 years of age. Indeed, we were generally unable to compare our results with those of previous reference interval studies that used the same analyser because the reference individual selection processes in these studies differed from ours. Nikulshin et al excluded patients without haematological disorders and tumours; however, they did not apply any other exclusion criteria to select healthy reference individuals. ${ }^{13}$ Oh et al and Kim $e t$ al included those who underwent regular medical check-ups but likewise did not use any additional exclusion criteria when selecting their healthy individuals. ${ }^{914}$ These approaches are common in many studies that investigate reference intervals; however, they carry the risk of including non-healthy individuals. We selected healthy reference individuals by using a stringent set of exclusion criteria from a large parent population, and established reference intervals for 26 research parameters along with 18 routine CBC components. As such, our data ought to be reliable and easily applicable to other laboratories after a proper validation process.

Our study was unique in the following aspects: (1) we selected healthy reference individuals based on stringent exclusion criteria; (2) the study was performed at a single centre, which helped minimise possible institutional or instrument-dependent bias; (3) the process of establishing reference intervals followed the CLSI guideline; and (4) we not only provided reference intervals, but also determined the necessity of sex-specific intervals for relevant parameters.

A possible limitation of this study was that we did not evaluate all the exclusion criteria suggested in the relevant CLSI guideline. ${ }^{1}$ Blood donation, lactation, obesity, occupation and the use of oral contraceptives, all of which are listed as possible exclusion criteria in the CLSI guideline, may affect CBC results. However, these were not considered exclusion criteria in our study owing to the lack of relevant data; ${ }^{1}$ moreover, such factors are inferred to have a minimal impact on the CBC parameters of individuals undergoing regular health check-ups. Instead, our exclusion criteria did encompass other rules that were not directly mentioned in the CLSI guideline but could nevertheless have influenced our test results, such as endoscopy/colonoscopy data, imaging studies and urinalysis. Of note, the Reed method was used to exclude extreme outliers that could have originated from non-healthy individuals. ${ }^{16}$ Another limitation is that we were unable to evaluate some parameters that were found to be clinically significant in previous studies, such as reticulocyte-associated parameters (eg, $\mathrm{CHr}$ ), owing to the lack of availability of the relevant data. ${ }^{11}$ The measurement of such parameters require additional reagents, which precluded their evaluation from the outset because we used an indirect, a posteriori sampling technique. An a priori approach may be helpful for providing reference intervals for such parameters. Lastly, the age range of our study population was relatively narrow, and the measured values may not have followed a normal distribution. Modelling method such as the Generalized Models for Location, Scale and Shape can help to address this issue by estimating percentiles.

In summary, we established a reliable set of reference intervals for 18 routine CBC components and 26 researchspecific parameters in Korean adults by selecting a large number of healthy reference individuals using stringent criteria. Our study showed that, even though most parameters showed statistically significant differences between men and women, none of the evaluated parameters, except for two that are RBC-related, required separate reference intervals for each sex. As such, our findings may have the capacity to serve as a model precedent for establishing more reliable reference intervals for research parameters during clinical laboratory testing.

Acknowledgements Statistical review was performed by JH.

Contributors MK was involved in conceptualising the study. Data curation was conducted by $\mathrm{KJ}$, JL and $\mathrm{JH}$. Formal analysis was performed by $\mathrm{KJ}$ and $\mathrm{JH}$. Investigation was carried by J-sL, H-SK, HJK and YKL. KJ wrote original draft and MK led the effort to supervision, write review and editing the final draft.

Funding The authors have not declared a specific grant for this research from any funding agency in the public, commercial or not-for-profit sectors.

Competing interests None declared.

Patient consent for publication Not required.

Ethics approval The use of patient data was approved by the Institutional Review Board of Hallym University Sacred Heart Hospital (approval no.: HALLYM 2018-12025). The requirement for written informed consent was waived by the board. The clinical data of patients was processed under anonymity and confidentiality.

Provenance and peer review Not commissioned; externally peer reviewed.

Data availability statement Data are available from the authors upon request.

Open access This is an open access article distributed in accordance with the Creative Commons Attribution Non Commercial (CC BY-NC 4.0) license, which permits others to distribute, remix, adapt, build upon this work non-commercially, and license their derivative works on different terms, provided the original work is properly cited, appropriate credit is given, any changes made indicated, and the use is non-commercial. See: http://creativecommons.org/licenses/by-nc/4.0/.

ORCID iD

Miyoung Kim http://orcid.org/0000-0002-8903-5044

\section{REFERENCES}

1 Clinical and Laboratory Standards Institute. Defining, establishing, and verifying reference intervals in the clinical laboratory; Approved Guideline-Third edition. EP EP28-A3. Wayne,PA: Clinical and Laboratory Standards Institute, 2010.

2 Adeli K, Higgins V, Nieuwesteeg M, et al. Biochemical marker reference values across pediatric, adult, and geriatric ages: establishment of robust pediatric and adult reference intervals on 
the basis of the Canadian Health Measures Survey. Clin Chem 2015;61:1049-62.

3 Tsang CW, Lazarus R, Smith W, et al. Hematological indices in an older population sample: derivation of healthy reference values. Clin Chem 1998:44:96-101.

4 Ridefelt $\mathrm{P}$, Hellberg D, Aldrimer M, et al. Estimating reliable paediatric reference intervals in clinical chemistry and haematology. Acta Paediatr 2014;103:10-15.

5 Golubeva V, Mikhalevich J, Novikova J, et al. Novel cell population data from a haematology analyzer can predict timing and efficiency of stem cell transplantation. Transfus Apher Sci 2014;50:39-45.

6 Buttarello M, Plebani M. Automated blood cell counts: state of the art. Am J Clin Pathol 2008;130:104-16.

7 Park SH, Park CJ, Lee BR, et al. Establishment of age- and genderspecific reference ranges for 36 routine and 57 cell population data items in a new automated blood cell analyzer, Sysmex XN-2000. Ann Lab Med 2016;36:244-9.

8 Semmelrock MJ, Raggam RB, Amrein K, et al. Reticulocyte hemoglobin content allows early and reliable detection of functional iron deficiency in blood donors. Clin Chim Acta 2012;413:678-82.

$9 \mathrm{Kim}$ MJ, Park PW, Seo YH, et al. Reference intervals for platelet parameters in Korean adults using ADVIA 2120. Ann Lab Med 2013;33:364-6.

$10 \mathrm{Kim} \mathrm{HK}, \mathrm{Kim} \mathrm{JE}$, Ham CK, et al. Prognostic value of platelet indices as determined by ADVIA 120 in patients suspected of having disseminated intravascular coagulation. Int J Lab Hematol 2008;30:117-23.
11 Enko D, Wallner F, von-Goedecke A, et al. The impact of an algorithm-guided management of preoperative anemia in perioperative hemoglobin level and transfusion of major orthopedic surgery patients. Anemia 2013;2013:641876

12 Rocco V, Castelli C, Fumi M, et al. The diagnostic use of ADVIA 2120 Siemens and an "APL criteria" can help to reduce the rate of early death in the APL. Int $J$ Lab Hematol 2019;41:124-32.

13 Nikulshin S, Tolstikova I, Bartule A, et al. Intracellular neutrophil myeloperoxidase level in pediatric patients: Significant age and gender variability. Int J Lab Hematol 2015;37:120-4.

14 A-C O, Lee JK, Hong JY, et al. Utilization of mean peroxidase index for discrimination of pseudoneutropenia. Lab Med Online 2013;3:1-5.

15 Lee EJ, Kim M, Lee E, et al. A comparison of complete blood count reference intervals in healthy elderly vs. younger Korean adults: a large population study. Clin Chem Lab Med 2019;57:716-29.

16 Horowitz G, Jones G, Rifai N. Establishment and use of reference intervals. In: Tietz textbook of clinical chemistry and molecular diagnostics. 6th edn. St Louis,Missouri: Elsevier, 2018: 185-6.

17 Reed AH, Henry RJ, Mason WB. Influence of statistical method used on the resulting estimate of normal range. Clin Chem 1971;17:275-84.

18 Harris EK, Boyd JC. On dividing reference data into subgroups to produce separate reference ranges. Clin Chem 1990;36:265-70.

19 Horowitz G, Jones G, Rifai N. Establishment and use of reference intervals. In: Tietz textbook of clinical chemistry and molecular diagnostics. 6th ed. St Louis,Missouri: Elsevier, 2018: 1-181. 